\title{
Associations between dairy consumption and common carotid intima media thickness in adults at risk of cardiovascular disease
}

The association between dairy consumption and cardiovascular disease (CVD) risk markers is controversial. Current evidence suggests an inverse association with either high milk consumption and lower risk of stroke development ${ }^{(1)}$ or overall dairy consumption and lower risk of coronary heart disease ${ }^{(2)}$. However, very little is known about the effects of dairy product consumption on novel risk markers of CVD, such as carotid intima media thickness (CIMT), a surrogate marker of atherosclerotic plaque development ${ }^{(3)}$.

In a subgroup of the RESET intervention study (REplacement of SaturatEd fat in dairy on Total cholesterol; NCT02089035), we determined the relationship between the amount and type of dairy products with novel and established CVD risk biomarkers. Baseline data on blood lipids, blood pressure and mean common carotid intima media thickness (CCA-IMT), using a B-mode ultrasound machine, were evaluated in 46 subjects (mean age 52 (sd 13) years; BMI 26 (sd 3.4) kg/m²; $\mathrm{n}=27$ men and $\mathrm{n}=19$ women) with an increased risk of CVD.

Participants were asked to complete a semi-quantitative food-frequency questionnaire (EPIC-Norfolk FFQ v.6) in order to determine dairy intake prior to starting the study. Correlations between dairy intake and CVD risk biomarkers were assessed using Spearman's rank coefficient of correlations (SPSS Inc., v.22).

\begin{tabular}{|c|c|c|c|c|c|c|}
\hline & \multicolumn{2}{|l|}{ Total } & \multicolumn{2}{|l|}{ Males } & \multicolumn{2}{|c|}{ Females } \\
\hline & Mean & SD & Mean & SD & Mean & SD \\
\hline Age (years) & 52 & 13 & 53 & 12 & 50 & 15 \\
\hline BMI $\left(\mathrm{kg} / \mathrm{m}^{2}\right)$ & $26 \cdot 0$ & $3 \cdot 4$ & $26 \cdot 6$ & $2 \cdot 6$ & $25 \cdot 9$ & $4 \cdot 4$ \\
\hline Systolic blood pressure (mmHg) & 129 & 13 & 131 & 9 & 125 & 13 \\
\hline Diastolic blood pressure $(\mathrm{mmHg})$ & 76 & 7 & 77 & 7 & 73 & 7 \\
\hline Total Cholesterol (mmol/L) & $6 \cdot 0$ & $1 \cdot 0$ & $5 \cdot 4$ & $0 \cdot 8$ & $6 \cdot 0$ & $1 \cdot 0$ \\
\hline LDL Cholesterol (mmol/L) & $3 \cdot 6$ & $0 \cdot 8$ & $3 \cdot 5$ & $0 \cdot 8$ & $3 \cdot 7$ & 0.9 \\
\hline Mean CCA-IMT (mm) & $0 \cdot 59$ & $0 \cdot 04$ & $0 \cdot 6$ & $0 \cdot 1$ & $0 \cdot 6$ & $0 \cdot 1$ \\
\hline Total Dairy consumption (g/d) & $379 \cdot 9$ & $188 \cdot 4$ & $384 \cdot 7$ & $165 \cdot 8$ & $372 \cdot 9$ & $221 \cdot 2$ \\
\hline Total Milk consumption (g/d) & $324 \cdot 5$ & $174 \cdot 2$ & $346 \cdot 9$ & $157 \cdot 8$ & $292 \cdot 6$ & $195 \cdot 2$ \\
\hline Total Cheese consumption (g/d) & $5 \cdot 2$ & $4 \cdot 2$ & 4.4 & 1.8 & $6 \cdot 3$ & $6 \cdot 1$ \\
\hline Total Yogurt consumption (g/d) & $19 \cdot 7$ & $22 \cdot 2$ & $13 \cdot 3$ & $17 \cdot 6$ & $28 \cdot 9$ & $25 \cdot 1$ \\
\hline Total Butter consumption $(\mathrm{g} / \mathrm{d})$ & $4 \cdot 1$ & $6 \cdot 3$ & 3.4 & $5 \cdot 3$ & $5 \cdot 5$ & $7 \cdot 7$ \\
\hline
\end{tabular}

Overall, the present baseline data showed no significant association between total dairy products, milk, cheese, butter or yogurt intake and CCA-IMT or other CVD biomarkers $(\mathrm{P}>0.05)$.

Although no relationship between dairy consumption with CIMT and other CVD factors was found, it remains necessary to conduct further studies to confirm our findings in healthy and at risk populations.

This research was supported by the Medical Research Council (MR/K020218/1), Arla Foods and AarhusKarlshamn (AAK) UK.

1. Elwood PC, et al. (2012) Lipids 45: 925-39.

2. Dalmeijer GW, et al. (2012) Int J Cardiol 167: 925-29.

3. de Groot E, et al. (2004) Circulation 109: III33-III38. 